\title{
Fortalezas familiares de los estudiantes universitarios: un acercamiento binacional
}

\section{Family strengths of college students: a binational approach}

DOI: $10.46932 / \mathrm{sfjdv2n2-186}$

Received in: March 1st, 2021

Accepted in: May 30th, 2021

\author{
M.T.F. Ana Elda Garay Burciaga \\ Docente de Tiempo Completo de la Facultad de Trabajo Social \\ De la Universidad Juárez del Estado de Durango, México.
}

M.C. Ma. Elena Martínez Jiménez

Docente de Tiempo Completo de la Facultad de Trabajo Social

De la Universidad Juárez del Estado de Durango, México.

\section{M.C. María Eugenia Pérez Herrera}

Docente de Tiempo Completo de la Facultad de Trabajo Social De la Universidad Juárez del Estado de Durango, México.

\section{RESUMEN}

El presente artículo da a conocer los resultados de la investigación denominada Fortalezas familiares de los estudiantes universitarios: un acercamiento binacional, la cual se desarrolló mediante un convenio entre la Universidad Juárez del Estado de Durango y la Universidad de Regina en Canadá. Ambos a través de las facultades de Trabajo Social para lo cual se desarrolla una intervención por parte de las investigadores Elena Martínez Jiménez y M.T.S María Eugenia Pérez Herrera, Dra. Judy White y Dr. Miguel Sánchez, los objetivos fueron 1.- Realizar un diagnóstico de las fortalezas familiares en una muestra de hogares de estudiantes de Trabajo Social de México y Canadá, 2.- Realizar un estudio comparativo de las FF entre las universidades participantes 3.- Diseñar una propuesta de intervención que permita potencializar las características familiares en pro de sus integrante, el abordaje se realizó a través de una metodología cuantitativa para lo cual se aplicó el Inventario de Fortalezas de las Familias de Estudiantes Universitarios México-Canadienses, siendo Dimensión 1. Imagen positiva de SI, Dimensión 2. Amor, formación y estrategia, Dimensión 3. Reglas y límites necesarios, Dimensión 4. Convivencia armónica, Dimensión 5. Liderazgo parental, Dimensión 6. Influencia educadora, Dimensión 7. Estereotipos o creencias de género siendo esta ultima una adaptación de los investigadores. se analizaron con el paquete estadístico SPSS V16, con los siguientes resultados: La población participante 93 en total pertenecen a los primeros tres semestres de la formación, en cuanto a la imagen positiva de sí misma (o) en mí familia se mantiene el rango más alto sosteniendo que si lo poseen, con relación a la dimensión Amor, origen y acción se encuentra de manera significativa la respuesta de que la familia tiene esta categoría integrada y una aportación importante es la dimensión de Roles y estereotipos de género la cual dentro de este estudio se presentaron elementos que permiten identificar que existen algunas ideas de violencia en donde creen que "el ser hombre es mejor que ser mujer" por lo que se hace necesaria la intensificación del trabajo en esta área.

Palabras clave: Fortalezas familiares, familia, genero. 


\begin{abstract}
This article presents the results of the research called Family strengths of university students: a binational approach, which was developed through an agreement between the Universidad Juárez del Estado de Durango and the University of Regina in Canada. Both through the faculties of Social Work for which an intervention is developed by the researchers Elena Martinez Jimenez and M.T.S Maria Eugenia Perez Herrera, Dr. Judy White and Dr. Miguel Sanchez, the objectives were 1. - To design an intervention proposal that allows to potentiate the family characteristics in favor of its members, the approach was carried out through a quantitative methodology for which the Inventory of Strengths of the Families of Mexican-Canadian University Students was applied, being Dimension 1. Positive image of the IS, Dimension 2. Parental leadership, Dimension 6. Educator influence, Dimension 7. Gender stereotypes or beliefs, the latter being an adaptation of the researchers. were analyzed with the SPSS V16 statistical package, with the following results: The participating population 93 in total belong to the first three semesters of training, as for the positive self-image (or) in my family remains the highest rank holding that if they possess it, in relation to the dimension Love, In relation to the dimension Love, origin and action, the response that the family has this category integrated is significant, and an important contribution is the dimension of Gender roles and stereotypes, which in this study presented elements that allow identifying that there are some ideas of violence where they believe that "being a man is better than being a woman", so it is necessary to intensify the work in this area.
\end{abstract}

Key words: Family strengths, family, gender.

\title{
1 INTRODUCCIÓN
}

Para hablar de las familias es necesario puntualizarla desde el cambio, en la concepción de este grupo de personas, se hace énfasis en lo planteado por el Dr. Raymundo Macías, quien considera que "es un grupo de adscripción natural de seres humanos con o sin lazos de consanguinidad, de pertenencia primaria y que conviven habitualmente bajo el mismo techo, que comparten la escasez o la abundancia de sus recursos de subsistencia y servicios y que, al menos en alguna etapa de su ciclo vital, incluye dos o más generaciones", si se analiza se integran algunos elementos que llevan a la concepción de las familias muy diferente de cómo se conocía como el modelo tradicional, el cual se ha modificado por situaciones sociales y legales según lo retoma la Dra Eleni Galatsopoulou (2015)

\section{ANTECEDENTES}

Fortalezas familiares

Rosario Esteinou (2010), en su libro: "Construyendo relaciones y fortalezas familiares. Un panorama internacional" señala que quienes establecen que "Los primeros estudios sobre fortalezas de las familias surgieron en los años treinta en Estados Unidos con el análisis de Woodhouse (1930) de 250 familias durante la época de la Gran Depresión.

Posteriormente siguió, el de Otto, sobre las fortalezas de las familias a principios de la década de los sesenta (Gabler y Otto, 1964; Otto, 1962, 1963). 
No es sino hasta la década de los setenta que la perspectiva sobre las fortalezas de las familias se empezó a desarrollar de manera más sistemática y consistente, cuando Nick Stinnett empezó su trabajo en la Universidad Estatal de Oklahoma en 1974 y subsecuentemente en la Universidad de Nebraska, en 1977. Stinnett, DeFrain y muchos colegas empezaron entonces a publicar una serie de artículos y libros sobre este tema (Stinnett y Sauer, 1977; Casas y otros, 1984; Stinnett y DeFrain, 1985; DeFrain, DeFrain y Lepard, 1994; Stinnett y O`Donell, 1996; Xie, DeFrain, Meredith y Combs, 1996; DeFrain y Stin- nett, 2002; Olson y DeFrain, 2006)” (Esteinou, 2010, pág. 11).

\section{PLANTEAMIENTO GENERAL}

Conocer los aspectos que se han venido investigando con relación a las fortalezas de las familia es primordial, ya que los integrantes han venido sorteando crisis a lo largo de su desarrollo; con ello ha venido enfrentando retos y desafíos ante las vicisitudes de la vida, para ello, conocer las diferentes políticas de desarrollo permite que la familia tenga la oportunidad de crecer y de desarrollarse en el mismo contexto donde se desenvuelve, pero con otra perspectiva de solución de conflictos dentro del contexto familiar.

Ante estos argumentos de las situaciones actuales que tienen que enfrentar las familias emerge la siguiente pregunta de investigación.

¿Cuáles son las fortalezas familiares en la estructura y funcionamiento en una muestra de hogares de estudiantes de Trabajo social de México y Canadá?

De este planteamiento surgen los siguientes objetivos generales

- Realizar un diagnóstico de las fortalezas familiares en una muestra de hogares de estudiantes de Trabajo Social de México y Canadá,

- Realizar un estudio comparativo de las Fortalezas familiares entre las universidades participantes

- Diseñar una propuesta de intervención que permita potencializar las características familiares en pro de sus integrantes

Dando los elementos para la generación de los siguientes Objetivos particulares Identificar las fortalezas predominantes en las familias.

- Señalar las dimensiones menos significativas como áreas de oportunidad en la familia.

- Conocer la estructura de las familias participantes.

- Detectar las fases del ciclo vital por las que transitan las familias.

- Señalar el tipo de familia predominante en la muestra.

- Mencionar los sucesos vitales afrontados por las familias encuestadas. 
- Identificar las semejanzas y diferencias de las fortalezas y/o áreas de oportunidad en las diferentes universidades participantes.

- Identificar los elementos fundamentales para elaborar una propuesta de intervención socioeducativa

\section{EL MARCO TEÓRICO}

La investigación planea retomar dos enfoques teóricos básicos por un lado la Teoría General de los Sistemas; la cual proponer una nueva disciplina científica, su tema es la formulación de principios válidos para "sistemas" en general, sea cual fuere la naturaleza de sus elementos componentes y las relaciones o "fuerzas" reinantes entre ellos.

Impulsar una terminología general que permita describir las características, funciones y comportamientos sistémicos; y sus principios básicos son: sistema, sistema cerrado, sistema abierto, totalidad o no sumatividad, circularidad, equifinalidad, entropía, homeostasis, morfogénesis, morfostasis, negentropía, sinergia (Bertalanffy, 2007).

Otro de los enfoques esta planteado desde la Teoría Ecológica

Busca la Interacción entre sistemas y contextos identificando recursos y fortalezas que existen en esas interacciones y en cada uno de los sistemas, desde los más próximos a los más distantes. Existe una interrelación entre los micro, meso y exosistemas en relación con las personas, así como la influencia de estos en ellas. Teniendo como principios básicos que los ambientes naturales son la principal fuente de influencia sobre la conducta humana, con lo cual la observación en ambientes de laboratorio o situaciones clínicas nos ofrece poco de la realidad humana. El desarrollo humano no es más que un cambio sostenido en el modo en que una persona interpreta, percibe, experimenta y se relaciona con su ambiente (Torrico y Cols. 2002).

Hoy día, las familias se han enfrentado a diversos cambios; como lo refieren Cangas y Moldes (2007):

"En las últimas décadas, el papel de la familia ha experimentado cambios radicales, ya que en siglos pasados era de tipo patriarcal o extensa. Actualmente han surgido nuevas fórmulas, entre las que se pueden incluir a las familias nucleares, las monoparentales, las ampliadas entre otros tipos" (citados por Álvarez 2011, pág. 1

Por todo lo anterior se puede decir que la familia es una unidad de análisis compleja estrechamente articulada con el entorno económico, social y su escenario histórico.

Su percepción está influenciada por la creencia cultural de que la familia nuclear es la natural, sana, adecuada y cualquier desvío supone algo inadecuado. 


\section{METODOLOGÍA}

Por su finalidad el estudio se propone como Investigación aplicada entendida como la utilización de los conocimientos en la práctica, para aplicarlos en provecho de los grupos que participan en esos procesos y en la sociedad en general, además del bagaje de nuevos conocimientos que enriquecen la disciplina. Ahora bien, el presente estudio pretende que el producto sea aplicable y de beneficio de la población que participa y a su vez que sea replicable en otros espacios.

Por su alcance temporal este es un estudio en cuanto a su alcance temporal es Seccional o sincrónica entendiendo por Tamayo (2003) como el que estudia la ciencia desde lo sincrónico; es decir no tiene en cuenta la dimensión temporal. Ya que el presente se realizará en un solo momento sin mantener una secuencia

En cuanto a su profundidad es correlacional ya que este tipo de investigación persigue fundamentalmente determinar el grado en el cual las variaciones de uno o varios factores. y la existencia y fuerza de la covariación normalmente se determina estadísticamente (Monjes 2011)

Por su amplitud. El estudio es desde la amplitud en donde los objetos de estudio clásicos de la Microsociología han sido la familia, los grupos de iguales, la pareja y las estructuras básicas de comportamiento en las relaciones. Se observa cómo de la interacción nacen las relaciones sociales, que van estructurando los distintos roles. (Araujo, 2018)

Por su naturaleza. La investigación es empírica pues su aporte al proceso de investigación es resultado fundamentalmente de la experiencia. Una de las cualidades de estos métodos es que posibilitan revelar las relaciones esenciales y las características fundamentales del objeto de estudio, accesibles a la detección sensoperceptual, a través de procedimientos prácticos con el objeto y diversos medios de estudio.

Tamayo (2014) sostiene que el tipo de muestreo aleatorio simple también conocido como el tipo de muestreo al azar, es decir, que cada uno de los individuos de una población tiene la misma posibilidad de ser elegido. Si no se cumple este requisito se dice que la muestra es viciada, por lo que si cada uno de los elementos de la población no tiene la misma posibilidad de ser elegido se habla entonces de una muestra viciada.

Técnicas será como lo establece Briones (2002) la de la encuesta social, ya que es uno de los tipos más utilizados de la investigación social cuantitativa, está definido como un método de obtención de información que utiliza preguntas orales o escritas, desde donde se plantea a un universo o muestra de personas, las cuales mantienen características requeridas en el problema de investigación.

Cabe señalar que la presente investigación se aplicado el instrumento. A continuación, se describen brevemente el instrumento: 
El Inventario de Fortalezas de las Familias de Estudiantes Universitarios México-canadienses, tiene como Objetivo La detección de fortalezas familiares permitirá a las familias darse cuenta de aquellas aspiraciones que tiene como familia, el grado en que las han alcanzado y aquellos focos vulnerables que aún no han sido atendidos. Asimismo, proporcionará datos importantes que impulsen pautas de acción para atender las familias ayudándolas a desarrollar sus áreas de oportunidad.

Consta de siete dimensiones, las cuales se describen a continuación: 1. Imagen positiva de si: se refiere a la percepción que tiene de sí misma la familia, en sus aspectos más relevantes como son la solución de problemas, la confianza, apoyo, estado de ánimo, satisfacción personal.

La dimensión 2. Amor, formación y estrategia se refiere a los motivos por los que se forma la familia, las demostraciones de afecto que se tienen, la frecuencia en sus manifestaciones afectivas.

La dimensión 3. Reglas y límites necesarios se refiere a la identificación de existencia de reglas, el grado de flexibilidad de las mismas, la capacidad de la familia de negociar. Responsabilidades y respecto a funciones familiares.

La dimensión 4. Convivencia armónica se refiere a la organización de actividades familiares, su frecuencia, maneras de vivir, relaciones interpersonales, tolerancia, así como temas y estilo de conversación.

La dimensión 5. Liderazgo parental. Influencia de la figura de padre, cuidador o tutor en la toma y coordinación de decisiones.

La dimensión 6. Influencia educadora se refiere a la actitud pro-social en familia, fomento de valores familiares, respeto a la legalidad, relación entre bienestar familiar, bienestar espiritual y bienestar comunitario.

La dimensión 7. Estereotipos o creencias de género mide el grado de aceptación hacia las concepciones culturales sobre los hombres y las mujeres. Roles tradicionales dentro del hogar, roles tradicionales sobre la mujer y roles tradicionales sobre el hombre.

\section{RESULTADOS}

A continuación, se dan a conocer los resultados obtenidos de la aplicación a 97 participantes jóvenes estudiantes de la Licenciatura en Trabajo Social de diversas unidades académicas del México como la universidad Autónoma de Guadalajara. Universidad Autónoma de Colima, Universidad Autónoma de Sinaloa campus Mazatlán y Culiacán, Universidad Autónoma de Hidalgo, Universidad Autónoma de Nuevo León, Universidad veracruzana y Universidad Juárez del Estado de Durango y la Universidad de Regina en Canadá. 
Se puede rescatar que se trata de una población conformada por el $88.04 \%$ por mujeres y el $11.96 \%$ por hombres. De los cuales se encuentran cursando los tres primeros semestres

Tabla 1 semestre que cursa la población participante

\begin{tabular}{ll}
\hline Semestre & Porcentaje \\
\hline Primer semestre & $15.05 \%$ \\
\hline Segundo semestre & $39.78 \%$ \\
\hline Tercer semestre & $45.16 \%$ \\
\hline
\end{tabular}

Es necesario resaltar que la población que formo parte del estudio se encuentra en las siguientes edades:

Tabla 2 edad de los participantes

\begin{tabular}{ll}
\hline Edad & Porcentaje \\
\hline 17 a 19 años de edad & $63.37 \%$ \\
\hline 20 a 22 años de edad & $28.88 \%$ \\
\hline 23 y más años de edad & $10.75 \%$ \\
\hline
\end{tabular}

En donde se puede observar que la mayoría de quienes formaron parte de esa muestra cuentan con la edad de 17 a 19 años de edad, lo que hace referencia a la etapa de juventud en la cual se encuentran los participantes.

Con relación a imagen positiva de sí misma (o) en mí familia las respuestas más significativas son las relacionadas con la pregunta "Me siento bien cuando estoy en mi familia" reportando que un total de $82 \%$ de los entrevistados señalaron la opción de respuesta 3, la cual dice "Mi familia tiene esta categoría"

La dimensión 2. Amor, formación y estrategia la respuesta mas significativa es la de Amo a mi familia y quiero que sea feliz en donde obtuvo el 92\% de la respuesta 3 la cual es "Mi familia tiene esta categoría" y sostiene que el 8\% están desarrollando y aun pueden crecer en esto, esta categoría en la que más altos niveles de respuesta positiva presenta.

La dimensión 3. Reglas y límites necesarios refiere que el dato mas significativo se encuentra relacionado con la frase "Para hacer algo, todos debemos estar de acuerdo" en la cual el $10 \%$ sostiene que no la tienen, el $44 \%$ lo está trabajando , lo que hace significativo que para más de la mitad de los encuestados no se sienten tomados en cuenta en la toma de decisiones, por el contrario en la frase "Respeto a los integrantes de mi familia" el $81 \%$ de los participantes sostienen que esto es algo con lo que cuenta su familia.

Con relación a la dimensión 4. Convivencia armónica en cuanto a si tienen problemas se hablan el $46 \%$ está trabajando en eso y el $8 \%$ no tienen esa condición lo que implica que aun en las familias es difícil dentro de la convivencia aclarar los problemas. otro de los elementos está relacionado con la frase 
Me siento cómodo con todos en mi familia en donde la respuesta del 75\% esta relacionada con el hecho de que en sus familias los miembros se sienten cómodos.

La dimensión 5. Liderazgo parental. En esta dimensión se observa una clara diferencia entre las figuras paternas. Por un lado, el 95\% manifiesta abiertamente la admiración que profesa hacia la madre y un $60 \%$ lo hace hacia el padre. Reflejando la notable diferencia entre el impacto que mantienen entre estas dos figuras. Otro elemento que marca un elemento positivo es la frase de "valoro los consejos de mi madre" que para el $81 \%$ de los participantes manifiesta que su familia cuenta con esta particularidad.

En cuanto a la dimensión 6. Influencia educadora, en la cual dos frases sobresalen en cuanto a sus resultados positivos por un lado "Inculcamos valores en casa" que para el $83 \%$ de los participantes se cuenta con este valor en su familia la frase "En familia respetamos la ley" la cual un 78\% sostiene que este elemento se practica dentro de la familia, por lo cual se fomenta los valores y el respeto como una actitud pro-social.

Finalmente, la dimensión 7. Estereotipos o creencias de género mantiene resultados de relevancia con relación a los roles, y las funciones de los hombres y las mujeres

Para el análisis se retoman tres respuestas significativas

Tabla 3 Emocionalmente la mujer posee mayor fortaleza que el hombre

\begin{tabular}{lr}
\hline Respuestas & \multicolumn{1}{c}{$\begin{array}{c}\text { Porcentaje } \\
\text { válido }\end{array}$} \\
\hline $\begin{array}{l}\text { 1. No la tenemos; no la } \\
\text { consideramos importante. }\end{array}$ & 36.0 \\
$\begin{array}{l}\text { 2. La estamos desarrollando, } \\
\text { aún podemos crecer en esto. }\end{array}$ & 26.2 \\
$\begin{array}{l}\text { 3. Mi familia tiene esta } \\
\text { categoría. }\end{array}$ & 35.4 \\
Total & 97.6 \\
\hline
\end{tabular}

Como se puede observar claramente se aprecia una disparidad muy corta , por lo que se puede inferir que al interior de las familias es una cuestión que se encuentra en proceso de evolución. Por otro lado, se encuentra

Tabla 4 Los hombres son superiores a las mujeres

\begin{tabular}{ll}
\hline Respuestas & Porcentaje \\
\hline 1. No la tenemos; no la consideramos importante. & 89.6 \\
2. La estamos desarrollando, aún podemos crecer en esto. & 4.3 \\
3. Mi familia tiene esta categoría. & 3.7 \\
& \\
\hline
\end{tabular}


Efectivamente estos participantes desmienten la superioridad e los hombres sobre las mujeres dentro de sus familias y un porcentaje menor dan como respuesta que se encuentran trabajando al respecto,

Por otro lado la pregunta relacionada con la supremacía jerárquica masculina se ve reflejada de la siguiente manera en las familias de los participantes

Tabla 5 El eje de la familia es indudablemente el padre

\begin{tabular}{ll}
\hline Respuestas & Porcentaje \\
\hline 1. No la tenemos; no la consideramos importante. & 48.2 \\
\hline 2. La estamos desarrollando, aún podemos crecer en esto. & 29.3 \\
\hline 3. Mi familia tiene esta categoría. & 19.5 \\
\hline
\end{tabular}

Se puede comparar claramente el hecho de que la mayoría de los participantes no consideran a los hombres como el eje fundamental. Lo que permite posicionar la idea de la coparticipación de los padres en la construcción y conducción de estas familias.

\section{CONCLUSIONES}

$\mathrm{Si}$ bien es cierto en este estudio la población participante mantiene elementos de fortaleza relacionados con la expresión de las emociones tanto positivas como negativas.

Un elemento que es por demás importante es el contexto en el cual se desarrolla la convivencia

La población participante 93 en total pertenecen a los primeros tres semestres de la formación

Imagen positiva de si misma (o) en mí familia se mantiene el rango mas alto con relación a que las familias si lo poseen

Con relación a la dimensión Amor, origen y acción se encuentra de manera significativa la respuesta de que la familia tiene esta categoría integrada

Es importante rescatar que no se puede circunscribir el impacto de las fortalezas familiares solo a los ámbitos particulares de los hogares, por el contrario, es un elemento de trascendencia social y comunitario en donde cada una de las fortalezas revisadas mantiene aplicabilidad en las relaciones y en la vida pública.

Tal es el caso del género y su impacto el cual dentro del estudio fue atendido desde la dimensión de Roles y estereotipos de género la cual dentro de este estudio se presentaron elementos que permiten identificar que existen algunas ideas de violencia en donde por ejemplo "el ser hombre es mejor que ser mujer" ya que algunos mantienen este pensamiento en su familia y otros no lo consideran así.

Actualmente la familia se enfatiza, hoy más que nunca, la necesidad de la educación en donde se puedan observar a futuro un cambio, en el compromiso por parte de todos y todas 


\section{BIBLIOGRAFÍA}

Álvarez Gayou, J.L. (2003). Cómo hacer investigación cualitativa. Fundamentos y metodología. México: Paidós.

Antuna, P. (2015). Guía de procedimientos para la presentación y elaboración de un proyecto de investigación de tesis. México. UJED

Arronte A., Beltran N, Correa E. Martínez M., Mendoza V., Rosado J., Sánchez M. Vargas L., Valdez F., (2015) Manual para la evaluación gerontológica integral de la comunidad (2 $2^{\mathrm{a}}$ Ed.) México. UNAM

Bertalanffy, Ludwig von (1968): Teoría general de los sistemas. México: Fondo de Cultura Económica, 1976.

Briones, G. (2002). Método y técnicas de investigación para las Ciencias Sociales. (4ª . Ed.). México: Trillas.

Bronfenbrenner, U. (1986). Ecology of the family as a context for human development: research perspectives. Developmental Psychology, 22(6), 723-742.

Campos, V. (2016). El concepto de familia en México: una revisión desde la mirada antropológica y demográfica. En R. Gutiérrez.

Clark-Carter, David. (2002). Investigación cuantitativa en Psicología. México: Oxford.

Esteinou R. (2010), Construyendo relaciones y fortalezas familiares. Un panorama internacional. H. Cámara De Diputados, LXI Legislatura Centro de Investigaciones y Estudios Superiores, en Antropología Social. Miguel Ángel PORRÚA, librero-editor 2010. ISBN 978-607-401-186

OMS (2002). Informe mundial sobre la violencia y la salud. Washington, DC: OPS

Rojas, R. (2004). El Proceso de la Investigación Científica. México. Editorial Trillas.

Tamayo y Tamayo (2001). El proceso de la investigación científica. México. Limusa.

Universidad Juárez del Estado de Durango (1957) Dirección de planeación y desarrollo académico. Modelo educativo de la Universidad Juárez del Estado De Durango. Julio 2006. Recuperado el 17 de marzo de 2021 Durango. Disponible en red http://famen.ujed.mx/doc/Modelo_educativo.Historia pdf. 